\title{
EFEITO DA MANIPULAÇÃO DE INIBIDOR SELETIVO DE RECAPTAÇÃO DE SEROTONINA SOBRE 0 CONSUMO DE DIETA HIPERLIPÍDICA E ATIVAÇÃO NEURONAL NO HIPOTÁLAMO DE RATAS ADULTAS
}

\section{${ }^{1,4}$ Amanda Alves Marcelino da Silva*, ${ }^{2}$ Mayara Matias Oliveira, ${ }^{3}$ Juliana Fonseca Nogueira Alves, ${ }^{4}$ Elton Gabriel Fernandes de Brito, ${ }^{1}$ Taisy Cinthia Ferro Cavalcante and ${ }^{2,5}$ Sandra Lopes de Souza}

1Programa de Pós-Graduação em Ciências da Saúde, Faculdade de Ciências Médicas, Universidade de Pernambuco - UPE, Campus Santo Amaro, Recife-PE, Brasil; ${ }^{2}$ Programa de Pós-graduação em Nutrição, Universidade Federal de Pernambuco - UFPE, Campus Recife, Recife-PE, Brasil; ${ }^{3}$ Programa de Pós-graduação em Educação Física e Saúde, Universidade Federal do Vale do São Francisco - UNIVASF, Campus Petrolina,

Petrolina-PE, Brasil; " Colegiado de Enfermagem, Universidade de Pernambuco - UPE, Campus Petrolina,

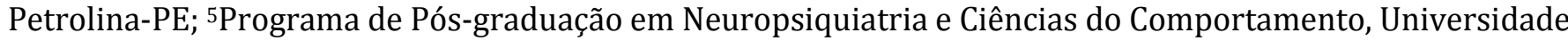
Federal de Pernambuco - UFPE, Campus Recife, Recife-PE, Brasil

\section{ARTICLE INFO}

\section{Article History:}

Received $17^{\text {th }}$ October, 2020

Received in revised form

$14^{\text {th }}$ November, 2020

Accepted $05^{\text {th }}$ December, 2020

Published online $30^{\text {th }}$ January, 2021

\section{Key Words:}

Dieta hiperlipídica; Fluoxetina;

Hipotálamo; ratas.

*Corresponding author:

Amanda Alves Marcelino da Silva,

\begin{abstract}
As doenças crônicas não transmissíveis são a principal causa de morbi-mortalidade do mundo. Atualmente, existem cerca de 1,6 bilhão de pessoas com sobrepeso e 650 milhões obesas. Provavelmente a principal causa disso, seja o consumo de dieta ricas em gorduras e carboidratos. O tratamento dessas doenças geralmente envolvi adjuvantes farmacológicos como os inibidores seletivos de recaptação de serotonina para reduzir o consumo alimentar ou até tratamentos mais invasivos como cirurgias (By-pass Gástrico) para redução da área de ingestão alimentar e saciedade. O objetivo desse trabalho foi investigar o efeito da inibição seletiva da recaptação de serotonina sobre a ingestão de dieta hiperlipídica e ativação de neurônios no núcleo arqueado e paraventricular em fêmeas adultas. Foram utilizadas ratas adultas, que sofreram aplicação de fluoxetina por 31 dias, e que consumiram dieta hiperlipídica durante 7 dias. As ratas apresentaram redução de peso corporal durante o consumo de dieta padrão. Houve aumento do consumo de dieta hiperlipídica após $24 \mathrm{~h}$ de exposição. E ao final dos 7 dias de exposição houve maior aumento ganho de peso corporal por parte do grupo fluoxetina. Portanto, a estimulação da via serotoninérgica é capaz de modular o controle da ingestão dieta hiperlipidica através da ativação de núcleos hipotalâmicos.
\end{abstract}

Copyright (C) 2020, Amanda Alves Marcelino da Silva et al. This is an open access article distributed under the Creative Commons Attribution License, which permits unrestricted use, distribution, and reproduction in any medium, provided the original work is properly cited.

Citation: Amanda Alves Marcelino da Silva, Mayara Matias Oliveira, Juliana Fonseca Nogueira Alves, Elton Gabriel Fernandes de Brito, Taisy Cinthia Ferro Cavalcante and Sandra Lopes de Souza. 2020. "Efeito da manipulação de inibidor seletivo de recaptação de serotonina sobre o consumo de dieta hiperlipídica e ativação neuronal no hipotálamo de ratas adultas", International Journal of Development Research, 11, (01), 43477-43483.

\section{INTRODUCTION}

Um desafio para os sistemas de saúde é o aumento da prevalência de doenças crônicas não transmissíveis, as quais repercutem de forma adversa nos padrões de morbimortalidade e nos custos dos serviços de saúde (WANG, MONTEIRO et al. 2002). O aumento de sobrepeso e obesidade tem assumido proporções de pandemia, sendo considerados hoje como graves problemas de saúde pública (ABRANTESL, LAMOUNIER et al. 2002).
De acordo com pesquisa, mais de 1,6 bilhão de adultos, acima de 18 anos apresentavam sobrepeso, destes 650 milhões eram obesos (Collaboration 2017). Esse aumento no número de indivíduos com sobrepeso e obesidade, está relacionado com um processo de mudança do padrão alimentar ocidental. Existe predomínio, em populações de países desenvolvidos ou em desenvolvimento, de consumo de dieta rica em gorduras (particularmente as de origem animal), sal, açúcar e alimentos refinados, além de reduzida ingestão de carboidratos complexos e fibras, o que frequentemente se denomina como 
"dieta ocidental" (Ferro Cavalcante, Lima da Silva et al. 2013). As dietas ricas em gordura e açucares simples são muito palatáveis e são capazes de promover alterações metabólicas estimulando o aumento da síntese de gordura, principalmente de triglicerídeos que se armazenam no tecido adiposo (Estadella, Oyama et al. 2004). Este acréscimo de tecido adiposo pode estimular a liberação de sinais de adiposidade como a leptina e a insulina (Fam, Morris et al. 2007, Ashino, Saito et al. 2011). Entretanto, a liberação acentuada desses peptídeos pode acarretar resistência de suas ações celulares e consequentemente seus efeitos se tornam reduzidos (Srinivasan, Katewa et al. 2006, Kirk, Samuelsson et al. 2009). Outra consequência será o aumento da ingestão alimentar, pois estes dois fatores atuam no estímulo da saciedade (Belgardt and Bruning 2010). Além disso, estes alimentos apresentam propriedades organolépticas, capazes de estimular vias de recompensa alimentar, ou seja, geram uma sensação de prazer (Lowe and Butryn 2007). Isto pode ser consequência do aumento na palatabilidade através do estímulo da atividade dos opióides endógenos (Tsujii, Nakai et al. 1986). Assim como a sinalização da via dopaminérgica dentro das áreas de recompensa também é alterada pela ingestão de alimentos densamente energéticos (Vucetic, Carlin et al. 2012).

Há mais de três décadas é reconhecido o papel da serotonina sobre o comportamento alimentar (Blundell 1977). A administração de inibidores seletivos de recaptação de serotonina (ISRS), precursores ou agonistas de receptores serotoninérgicos, de forma geral, inibem a ingestão alimentar por estimular a saciedade (um fenômeno homeostático) (Blundell 1984, Dourish 1995, Simansky 1996). A produção de serotonina (5-HT) encefálica localiza-se nos núcleos da rafe do tronco encefálico (Fuller and Wong 1990). A 5-HT é sintetizada a partir do aminoácido precursor triptofano, um aminoácido essencial. Presente na circulação plasmática ligado a albumina, formando o complexo triptofano-albumina (Curzon, Friedel et al. 1973). Para que esse aminoácido entre no sistema nervoso central é necessário atravessar a barreira hematoencefálica. O primeiro passo é se separar da albumina por dois mecanismos: pela interação entre o complexo triptofano-albumina e o glicocálice das células endoteliais da barreira e/ou por uma diminuição na taxa de fluxo sanguíneo (Smith, Momma et al. 1987, Pardridge and Fierer 1990). O triptofano é transportado através da barreira hematoencefálica por meio do transportador de aminoácidos tipo L (LAT1) (Christensen, Albritton et al. 1994, Matsuo, Tsukada et al. 2000) e torna-se disponível para a síntese de serotonina (Ruddick, Evans et al. 2006). Quando o triptofano penetra no soma do neurônio serotoninérgico, é imediatamente hidroxilado pela enzima triptofano hidroxilase-2 em 5hidroxitriptofano (5- HTP). Para essa reação é utilizado o oxigênio molecular $\left(\mathrm{O}_{2}\right)$ e o cofator tetrahidrobiopterina $\left(\mathrm{BH}_{4}\right)$ (composto sintetizado a partir do GTP) responsável por catalisar a hidroxilação (Ruddick, Evans et al. 2006). O 5-HTP é rapidamente convertido em 5-hidroxitriptamina (serotonina) através da enzima descarboxilase dos ácidos aromáticos, utilizando como co-fator o piridoxal-5-fosfato (P5P). A serotonina pode ser então: a) metabolizada pela flavoproteína monoamina-oxidase A (localizado na membrana mitocondrial) em 5- hidroxiacetaldeído (5-HIA) e em seguida no seu metabólito ácido 5-hidroxindolacético (5-HIAA) através da enzima aldeído desidrogenase. Após ser produzido, o 5-HIAA é excretado da célula por meio de um mecanismo dependente de energia, b) A 5-HT também pode ser empacotada em vesículas sinápticas, através da ação do transportador vesicular de monoamina tipo 2 (VMAT2), encontrado exclusivamente no $\mathrm{SNC}$, vinculado à proteína transportadora de ligação da serotonina (SBP) podendo ser liberado para o líquido extracelular por exocitose em um processo dependente de cálcio. c) A 5-HT quando liberada pode ser reciclada para o interior do neurônio pré-sináptico através da proteína de recaptação de serotonina (SERT) ou pode ser metabolizada a 5-HIAA por células pós-sinápticas contendo monoamina oxidase A (MAO A) (Fuller and Wong 1990, Erickson, Schafer et al. 1996, Wade, Chen et al. 1996, Ruddick, Evans et al. 2006, Siegel and Douard 2011, Fidalgo, Ivanov et al. 2012). Esse neurotransmissor possui sete famílias de receptores designados de $5 \mathrm{HT}_{1} \mathrm{R}-5 \mathrm{HT}_{7} \mathrm{R}$, subdivididas em 14 subtipos. Os principais receptores implicados na regulação homeostática da ingestão alimentar são o $5-\mathrm{HT}_{1 \mathrm{~B}}$ e o $5-\mathrm{HT}_{2 \mathrm{C}}$ (Heisler, Jobst et al. 2006). Neurônios NPY/AgRP e POMC/CART do núcleo arqueado do hipotálamo apresentam receptores $5-\mathrm{HT}_{1 \mathrm{~B}}$ e $5-\mathrm{HT}_{2 \mathrm{C}}$, que quando estimulados inibem a via orexigênica e estimulam a anorexigênica resultando em saciedade (Heisler, Jobst et al. 2006). Terminais serotoninérgicos fazem sinapse com neurônios que expressão POMC através do receptor $5-\mathrm{HT}_{2 \mathrm{C}}$, por outro lado através da ativação de 5-HT $1 \mathrm{~B}$ neurônios que expressam NPY e AgRP são inibidos assim como sua ação inibitória sobre POMC através de projeções GABAérgicas (Heisler, Jobst et al. 2006). Estes resultados indicam que a serotonina, no controle homeostático do balanço energético, promove saciedade por estimular neurônios anorexigênicos e inibir ao mesmo tempo os orexigênicos no núcleo arqueado do hipotálamo.

Em algumas situações, a serotonina pode exercer efeito oposto sobre o balanço energético, ou seja, estimular a ingestão de alimento. Essa ação ocorre através dos mecanismos do controle hedônico do consumo de alimentos palatáveis (Gray and Cooper 1996). Embora os mecanismos ainda sejam pouco conhecidos, acredita-se que essa ação pode ser indireta, através da modulação da serotonina sobre a neurotransmissão dopaminérgica (Alex and Pehek 2007). Essa hipótese se baseia no fato que a estimulação ou inibição de neurônios dos núcleos da rafe produz, respectivamente, aumento e diminuição na liberação da dopamina no nucleus accumbens (Yoshimoto and McBride 1992). Ademais a administração direta de serotonina dentro da área tegmentar ventral ou no nucleus accumbens, aumenta os níveis de dopamina extracelular (Guan and McBride 1989, Parsons and Justice 1993). Atualmente, existem evidências para o papel direto da serotonina sobre o controle do consumo de alimento palatável (Pratt, Blackstone et al. 2009). O nucleus accumbens contém elevada densidade de receptores 5- $\mathrm{HT}_{1 \mathrm{~B}}$ (Bruinvels, Palacios et al. 1993, O'Dell and Parsons 2004). Foi observado que a utilização de agonista (EMD 386088) do receptor 5HT-6 é capaz de aumentar o consumo de dieta palatável e de dieta padrão (Pratt, Blackstone et al. 2009). Com base nisso, este trabalho teve como objetivo investigar o efeito da inibição seletiva da recaptação de serotonina sobre a ingestão de dieta hiperlipídica e ativação de neurônios no núcleo arqueado e paraventricular em fêmeas adultas.

\section{METODOLOGIAS}

Animais e Droga: Todos os experimentos foram realizados de acordo com as recomendações do Comitê Brasileiro de Experimentação Animal (COBEA) e foram aprovados pelo Comitê de Ética em Experimentação Animal do Centro de 
Ciências Biológicas da Universidade Federal de Pernambuco $\left(\mathrm{n}^{\circ}\right.$ do processo 23076.037409/2011-64). Foram utilizados ratas da linhagem Wistar oriundos do Biotério de Criação do Departamento de Nutrição da Universidade Federal de Pernambuco, dos 110 aos 142 dias de vida, que serão mantidos em biotério invertido, ciclo claro-escuro $(12 \mathrm{~h} / 12 \mathrm{~h})$, com luzes acesas às $18 \mathrm{~h}$ e temperatura de $22^{\circ} \pm 1$, e em condições padrão de biotério água e ração (Labina Purina ${ }^{\circledR}$ ) ad libitum. Os grupos foram divididos segundo a manipulação farmacológica com inibidor seletivo de recaptação de serotonina (cloridrato de fluoxetina, $n=10$ ) ou salina (solução salina, $0,9 \% \mathrm{NaCl}$, $n=10$ ).

Manipulação Dietética e Peso Corporal: Foi realizado o consumo de dieta padrão por 22 dias (a cada 3 dias) e a partir do $25^{\circ}$ ao $31^{\circ}$ dia de manipulação farmacológica os animais foram expostos à dieta hiperlipídica $(4,37 \mathrm{Kcal} / \mathrm{g})$. A análise de consumo de dieta hiperlipídica no $1^{\circ}, 4^{\circ}, 7^{\circ}$ dias de exposição à dieta hiperlipídica. O ganho de peso corporal foi analisado ao longo do $1^{\circ}$ e $7^{\circ}$ dia de consumo de dieta hiperlipídica.

\section{Estímulo Alimentar e/ou Farmacológico para expressão da Proteína FOS}

Aos 142, os animais pertencentes aos grupos Fêmea-Salina, Fêmea-Fluoxetina, foram submetidos a estímulos específicos para estudo da expressão da proteína FOS. A proteína FOS pertence ao grupo de proteínas que funcionam como marcadores metabólicos de atividade neuronal (Dragunow and Faull 1989). Estes animais foram subdivididos em dois grupos de acordo com estímulo para expressão da proteína FOS: o grupo Fêmea-Salina $(n=5)$ foi formado por animais que sofreram aplicação crônica de cloridrato de fluoxetina e expostos apenas a dieta hiperlipídica por $90 \mathrm{~min}$; o grupo Fêmea-Fluoxetina $(\mathrm{n}=5)$, formado por animais que receberam aplicação de solução salina seguida de exposição de dieta hiperlipídica por $90 \mathrm{~min}$. Estes animais sofreram aplicação da última dose de cloridrato de fluoxetina às 7 da manhã seguida de privação alimentar por cinco horas.

Perfusão Transcardíaca: Os animais foram anestesiados com uma combinação de ketamina $(1 \mathrm{ml} / \mathrm{Kg})$ e xilazina $(0,1 \mathrm{ml} / \mathrm{kg})$, seguido da abertura da cavidade torácica para acesso ao ventrículo esquerdo e introdução da cânula para perfusão. Inicialmente foi infundido $150 \mathrm{ml}$ de solução salina $(\mathrm{NaCl}$, $0,9 \%$ ) a temperatura ambiente para remoção do sangue dos vasos. Seguida de infusão de $400 \mathrm{~mL}$ solução fixadora ( $4 \%$ de paraformoldeído, $\mathrm{pH} 7,4$, em $4^{\circ} \mathrm{C}$ ). Ao final da passagem do fixador, os encéfalos foram retirados do crânio e pós-fixados na mesma solução fixadora acrescida de sacarose $(20 \%)$ durante 4 horas. Após esse período, foram armazenados em solução crioprotetora (Tampão fosfato de sódio PBS acrescido de sacarose $30 \%$ ) até a realização dos cortes.

Imunohistoquímica contra a proteína Fos: Os cortes de uma série, contendo os núcleos hipotalâmicos foram processados para imunohistoquímica contra Fos pelo método da peroxidase. Para isso, após lavagens sucessivas com solução tampão, os cortes foram incubados com anticorpo primário anti-fos (1:10000) por 24 horas. Posteriormente, foram incubados em anticorpo secundário biotinilado (diluição 1:200) por 90 min. Após amplificação do sinal com o complexo avidina-biotina, a revelação foi realizada por diaminobenzidina. As marcações evidentes foram contadas, com auxílio de microscópio de luz, nas regiões selecionadas de todos os cortes.

Análise quantitativa de neurônios imunorreativos a proteína FOS em áreas do hipotálamo relacionadas com o comportamento alimentar : A identificação, delimitação e quantificação de neurônios serão realizadas a partir de fotomicrografias adquiridas por meio de uma câmera digital (Samsung SHC-410NAD) adaptada a um microscópio (Olympus BX50), e um computador (Funtech). A aquisição das imagens será realizada utilizando a objetiva $10 \mathrm{X}$ e com o auxílio do software TV Turner Application. Para a identificação e delimitação das áreas estudadas será utilizado como referência o Atlas Estereotáxico de Rato (PAXINOS e WATSON, 2005). As áreas escolhidas para este estudo serão: núcleo arqueado e paraventricular.

Análise estatística: Os dados de peso, consumo alimentar, ganho de peso corporal e número de células imunorreativas a proteína c-fos estão apresentados em média e erro padrão. Foi utilizado o ANOVA two way para análise dados de peso, consumo alimentar seguido do pós-teste de Bonferroni para comparar o efeito da aplicação crônica de fluoxetina. Na análise de ganho de peso corporal e avaliação da ativação de $\mathrm{c}$-fos foi utilizado o teste $\mathrm{t}$ de Student. Para todas as avaliações foi considerado $\mathrm{p}<0,05$. Todos dados foram analisados utilizando GraphPad Prism 5 software, versão 7 (GraphPad Inc., La Jolla, CA, USA).

\section{RESULTADOS}

Peso Corporal e Consumo de Dieta Padrão: Foi observado efeito do ISRS sobre o peso corporal $\left(\mathrm{F}_{1,160}=74,40, \mathrm{p}<0,0001\right)$ do $4^{\circ}$ ao $22^{\circ}$ dia de consumo de dieta padrão em fêmeas. Nesse gênero, o grupo fluoxetina (FF) apresentou redução do peso corporal quando comparado ao grupo salina (FS) no $1^{\circ}$ (FS= $223.60 \pm 2,37, \mathrm{n}=12 ; \mathrm{FF}=212.86 \pm 4.63 \mathrm{n}=10, \mathrm{p}>0,05), 4^{\circ}$ $(\mathrm{FS}=226.64 \pm 2,36, \mathrm{n}=12 ; \mathrm{FF}=213.84 \pm 3,95, \mathrm{n}=10, \mathrm{p}<0,05)$ e $22^{\circ}(\mathrm{FS}=239,42 \pm 2,96, \mathrm{n}=12 ; \mathrm{FF}=224.95 \pm 3,61, \mathrm{n}=10$ $\mathrm{p}<0,05)$ dias de tratamento. A fluoxetina promoveu efeito sobre o consumo de dieta padrão nas fêmeas $\left(\mathrm{F}_{(1,160)}=27,06\right.$, $\mathrm{p}<0,0001)$. O grupo fluoxetina apresentou redução no consumo alimentar no $1^{\circ}(\mathrm{FS}=8,32 \pm 0,38, \mathrm{n}=12 ; \mathrm{FF}=5,93 \pm$ $0,44, \mathrm{n}=10, \mathrm{p}>0,001)$ e no $4^{\circ}$ dia de análise ( $\mathrm{FS}=8,69 \pm 0,53$, $\mathrm{n}=12 ; \mathrm{FF}=6,49 \pm 0,27, \mathrm{n}=10, \mathrm{p}<0,001)$.

Consumo de Dieta Palatável e Percentual de Ganho de Peso por Fêmeas sob Efeito Crônico de ISRS: No estudo com fêmeas, o grupo fluoxetina apresentou aumento no consumo alimentar de 24 horas no $1^{\circ}(\mathrm{FS}=9,35 \pm 0,44, \mathrm{n}=10$; $\mathrm{FF}=9,99 \pm 0,34, \mathrm{n}=10, \mathrm{p}<0.0019)$ e redução no $7^{\circ}$ dia de exposição $(\mathrm{FS}=5,96 \pm 0,33, \mathrm{n}=10 ; \mathrm{FF}=5,51 \pm 0,42, \mathrm{n}=10$, $\mathrm{p}<0.015$ ) (Figura 2). Não foi observada diferença entre os grupos no $4^{\circ}$ dia $(\mathrm{FS}=7,40 \pm 0,98, \mathrm{n}=10 ; \mathrm{FF}=8,06 \pm 0,44$, $\mathrm{n}=10, \mathrm{p}=0,5513)$. A inibição seletiva crônica da recaptação de serotonina promoveu maior percentual de ganho de peso em fêmeas comparado aquele do grupo salina $(\mathrm{FS}=3,47 \pm 0,73$, $\mathrm{n}=10 ; \mathrm{FF}=6,01 \pm 0,84, \mathrm{n}=10 ; \mathrm{p}<0.034$ ) (Figura 3).

Ativação Neuronal em resposta ao ISRS e dieta hiperlipídica no núcleo arqueado e paraventricular do hipotálamo: A análise da ativação neuronal mostrou maior ativação das fêmeas tratadas com fluoxetina no núcleo arqueado (FF- ARC- 134, $75 \pm 2,93, \mathrm{n}=4$ ) quando comparadas as fêmeas salina (FS-101,50 $\pm 5,04, n=4, p=0,0013$ ). 

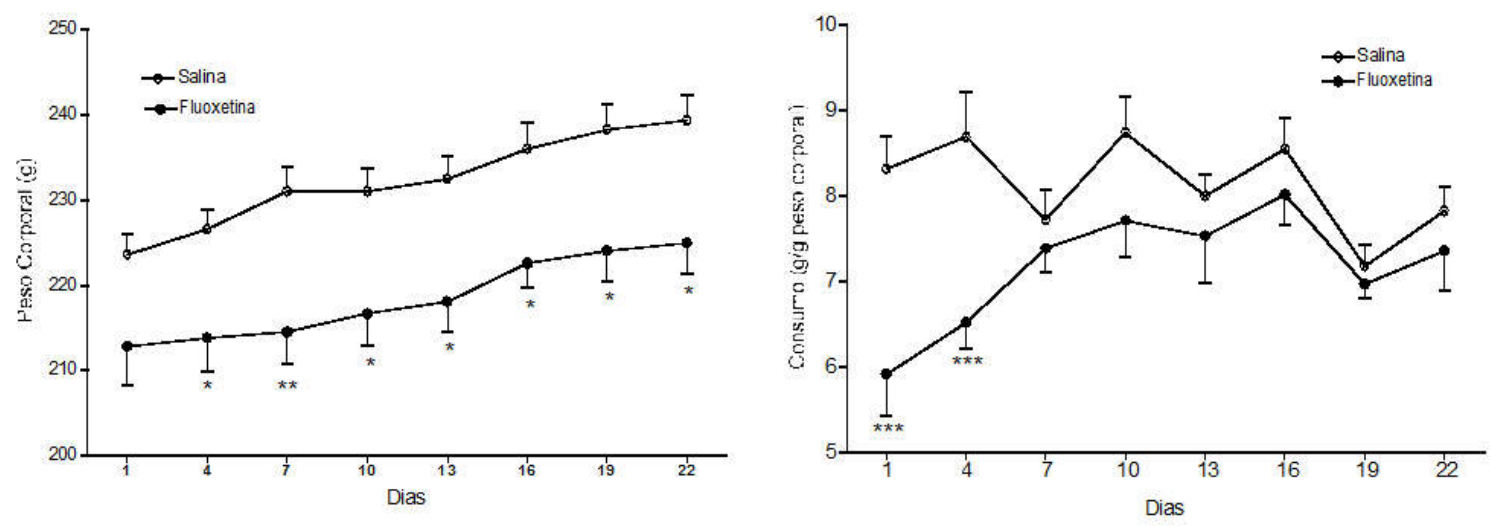

Figura 1. Peso corporal e consumo alimentar de dieta padrão em ratas manipuladas com inibidor seletivo de recaptação de serotonina durante 22 dias. Os dados foram representados em média e erro padrão. Grupos experimentais: Fêmea-Salina, FS, n=8; Fêmea-

Fluoxetina, FF, n=8 Peso Corporal de fêmeas, Consumo Alimentar de fêmeas. Foi utilizado o Two-way Anova, seguido de Bonferroni Test, $\mathbf{p}<0,05$

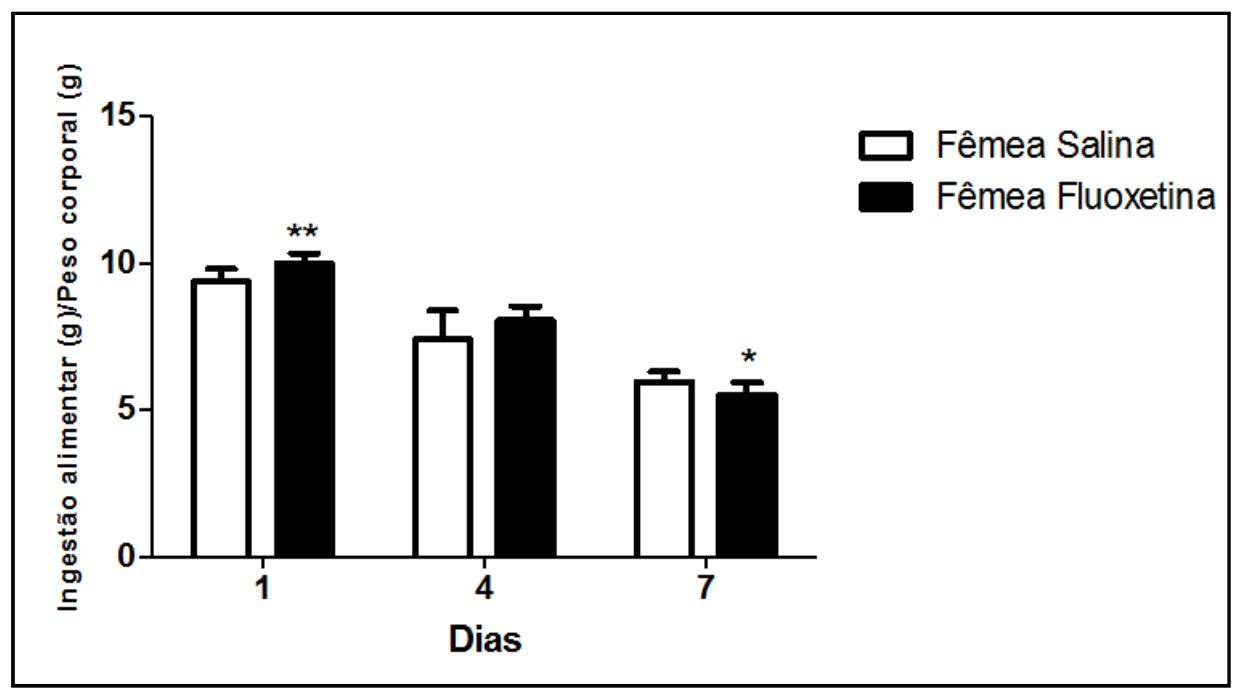

Figura 2. Consumo crônico de dieta HFHS por fêmeas, sob estimulação crônica com fluoxetina. Os dados foram representados em média e erro padrão. Grupos experimentais: Fêmea-Salina, n=10; Fêmea-Fluoxetina, n=10 (A) Consumo alimentar das fêmeas. Foi utilizado test $\mathbf{T}$ de Student, $\mathbf{p}<0.05$

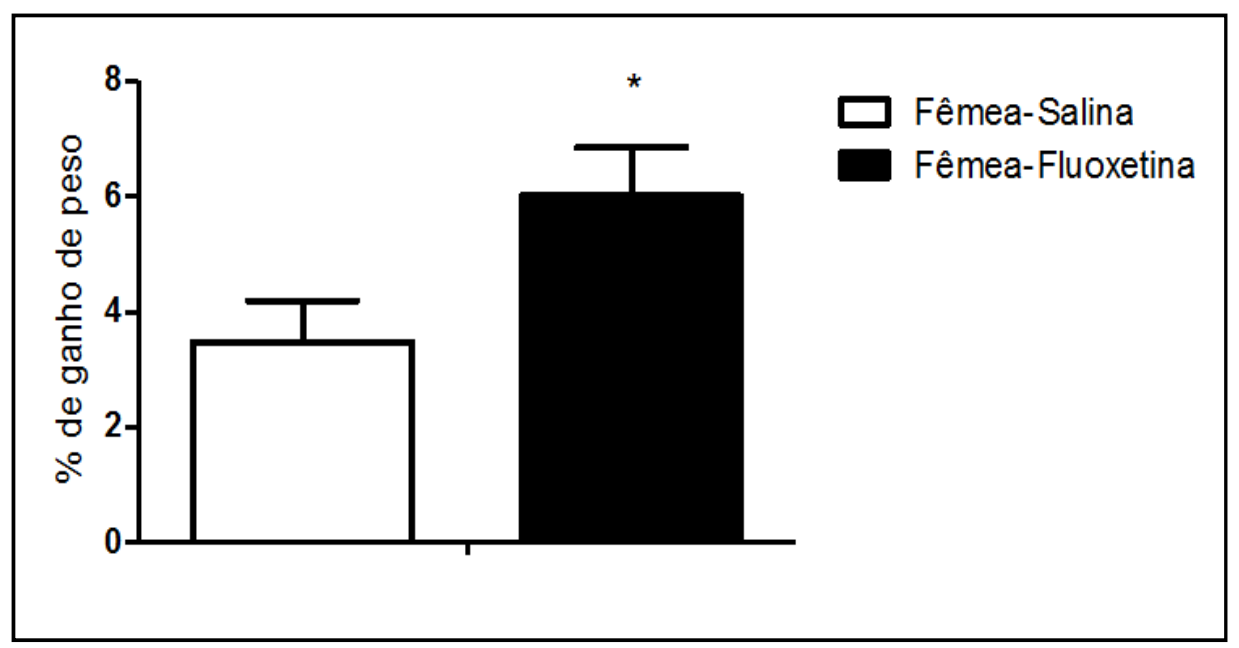

Figura 3. Percentual de ganho de peso após sete dias de dieta HFHS e aplicação crônica de inibidor seletivo de recaptação de serotonina. Foi utilizado teste $T$ de Student. Os dados foram representados em média e erro padrão. Os dados foram representados em média e erro padrão. Grupos experimentais: Fêmea-Salina, $n=10$; Fêmea-Fluoxetina, $n=10$

Enquanto, houve menor ativação do núcleo paraventricular nas fêmeas tratadas com fluoxetina (FF- 65,0 $\pm 2,8, \mathrm{n}=4)$ quando comparadas com as fêmeas salina (FS- 76,0 $\pm 2,10, n=4$, $\mathrm{p}=0,0200$ ) (Figura 4).

\section{DISCUSSÃo}

O presente trabalho investigou o efeito da inibição crônica seletiva da recaptação de serotonina sobre a ingestão de dieta 
rica em lipídios e glicídios. Para isto, foi utilizada a fluoxetina, uma droga psicoanálepticas pertencente à classe dos antidepressivos. A fluoxetina age bloqueando a proteína transportadora de recaptação de serotonina (SERT), e inibe o receptor pré-sináptico $5 \mathrm{HT}_{1 \mathrm{a}}$ (Sghendo and Mifsud 2012).

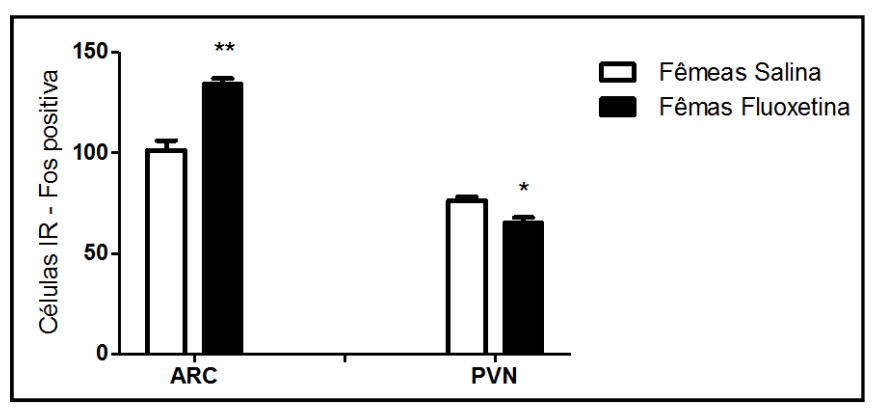

Figura 4. Ativação neuronal em resposta a aplicação de inibidor seletivo de recaptação de serotonina no núcleo arqueado e núcleo paraventricular de fêmeas que consumiram dieta hiperlipidica durante 22 dias. Os dados foram representados em média e erro padrão. Grupos experimentais: Fêmea-Salina, $n=4$; Fêmea-

Fluoxetina, $n=4$. Foi utilizado test $T$ de Student, $p<0.05$

A SERT é responsável pela captação da serotonina liberada na fenda sináptica após sua ação, enquanto que o receptor $5 \mathrm{HT}_{1 \mathrm{a}}$, funciona como autorregulador da liberação de serotonina (Sghendo and Mifsud 2012). Portanto, o efeito farmacológico final da fluoxetina é a potencialização da via serotoninérgica (Sghendo and Mifsud 2012). O presente estudo demonstrou que a inibição da recaptação da serotonina reduziu o consumo de dieta padrão e o peso corporal das fêmeas. A potencialização da via serotoninérgica afetou o peso corporal durante a ingestão de dieta padrão e promoveu redução de consumo durante os primeiros dias de aplicação. Os inibidores seletivos de recaptação da serotonina estão relacionados a perda de peso e redução do consumo alimentar (Gutierrez, Saracibar et al. 2002, Mennigen, Harris et al. 2009, Iniguez, Warren et al. 2010, Lauzurica, Garcia-Garcia et al. 2013). A redução do consumo pode ser explicada pela capacidade da serotonina promover saciedade, através de sua ação no núcleo arqueado do hipotálamo (Leibowitz and Alexander 1998), especialmente sobre o receptor $5 \mathrm{HT}_{2 \mathrm{C}}$. Estudo demonstrou que a exposição à fluoxetina durante duas semanas promoveu aumento na expressão de POMC (peptídeo anorexigênico) sem afetar a expressão de AgRP (Peptídeo orexigênico) no núcleo arqueado hipotalâmico (Churruca, Portillo et al. 2008).

Em outro estudo, foi observado que a fluoxetina reduz a ativação de células imunoreativas a NPY (peptídeo orexigênico) no núcleo paraventricular do hipotálamo, mas sem mudança de ativação no núcleo arqueado (Gutierrez, Saracibar et al. 2002). Um estudo demonstrou que a ação da fluoxetina é dependente dos estoques de serotonina encefálica, e que aplicada de forma crônica a fluoxetina é capaz de causar gradual desenssibilização do receptor $5 \mathrm{TH}_{1 \text { a }}$ pré-sináptico $(\mathrm{Li}$, Muma et al. 1996), o que corrobora e explica os resultados aqui apresentados. No presente estudo foi observado redução do consumo alimentar de dieta padrão sob efeito agudo da inibição de recaptação da serotonina. Nas fêmeas esta hipofagia se prolongou até o quarto dia de análise. A aplicação de fluoxetina durante 5 dias promove aumento da expressão de SERT nos núcleos da rafe em ambos os sexos (Lauzurica, Garcia-Garcia et al. 2013). A fluoxetina inicialmente promove elevação da serotonina na fenda sináptica como resultado da inibição da recaptação. Essa elevação de 5HT gera uma sensação de saciedade e consequentemente hipofagia. Como reação de feedback o neurônio sintetiza mais SERT (Lopez, Chalmers et al. 1994). Com o tempo, com reflexo de uma sustentada inibição da recaptação, a síntese de SERT pode sofrer um slow down, como resultado do decréscimo da transcrição do gene SERT (Lopez, Chalmers et al. 1994). Esse resultado é dependente da dose e do tempo de tratamento com a fluoxetina (Oliva, Uriguen et al. 2005). Outro fator importante é a relação da ação anorética da leptina dependente da sinalização serotoninérgica no núcleo arqueado do hipotálamo (Yadav, Oury et al. 2011). A leptina inibe a liberação de serotonina, e consequentemente reduz sua ação sobre o receptor 5HT1a pós-sináptico (Yadav, Oury et al. 2011). A ação da serotonina sobre o receptor 5HT1a póssináptico aumenta o consumo alimentar (Yadav, Oury et al. 2011). Essa ação da leptina resultaria em inibição da ingestão alimentar (Yadav, Oury et al. 2011). Porém, a aplicação crônica de fluoxetina reduz a circulação plasmática de leptina, prejudicando esse mecanismo de controle entre a leptina e a serotonina (Dryden, Brown et al. 1999).

A exposição crônica a fluoxetina e a dieta HFHS (sete dias) promoveu, nas fêmeas, aumento seguido de redução do consumo alimentar nas fêmeas. As fêmeas também apresentaram maior ganho de peso, entretanto oscilou no padrão de ingestão alimentar. A redução do consumo alimentar de fêmeas após sete dias de dieta palatável pode estar relacionada a ação do estrógeno na via de controle hipotalâmico do comportamento alimentar. $\mathrm{O}$ estrógeno atua através da redução da expressão do NPY no núcleo paraventricular do hipotálamo, o que reduz o consumo alimentar (Bonavera, Dube et al. 1994). O estradiol também diminui o efeito orexigênico do hormônio concentrador de melanina, por possivelmente reduzir sua sinalização (Messina, Boersma et al. 2006). Estudos são controversos quanto à ação da fluoxetina sobre o consumo de macronutrientes. Um estudo sobre o efeito da fluoxetina na ingestão dos macronutrientes concluiu que esse ISRS reduz o consumo de gordura e proteína, mas não a de carboidrato (Heisler, Kanarek et al. 1997, Heisler, Kanarek et al. 1999). Em outro estudo observou-se que mudanças nos níveis de serotonina no núcleo paraventricular do hipotálamo, aumentam o consumo de gordura e inibe o de carboidrato (Weiss, Rogacki et al. 1991).

\section{Conclusão}

A hiperestimulação do sistema serotoninérgico através da manipulação de ISRS foi capaz de aumentar capaz de aumentar o consumo de dieta rica em lipídios e o ganho de peso corporal em fêmeas. Além de aumentar a ativação neuronal no núcleo arqueado enquanto diminui no núcleo paraventricular. Portanto, a estimulação da via serotoninérgica é capaz de modular o controle da ingestão dieta hiperlipidica através da ativação de núcleos hipotalâmicos.

\section{Agradecimentos}

Os pesquisadores agradecem aos suportes financeiros da Coordenação de Aperfeiçoamento de Pessoal de Nível Superior (CAPES) e da Fundação de Amparo a Ciência e Tecnologia do Estado de Pernambuco (FACEPE - número de protocolo: 0496-4.05/14). Agradecemos também ao Laboratório de Imunopatologia Keiso Asami (LIKA) pelo apoio técnico metodológico e a Universidade Federal de Pernambuco. 
Conflito de interesse: Os autores não apresentam conflito de interesse.

\section{REFERÊNCIAS}

ABRANTESL, M. M., A. J. LAMOUNIER and A. E. COLOSIMO (2002). "Prevalência de sobrepeso e obesidade em crianças e adolescentes das regiões Sudeste e Nordeste. ." Jornal de Pediatria. Vol. 78, ( $\mathrm{N}^{\circ} 4$, ): p.335340 .

Alex, K. and E. Pehek (2007). "Pharmacologic mechanisms of serotonergic regulation of dopamine neurotransmission." Phamacol. Ther. 113(2): 296-320.

Ashino, N. G., K. N. Saito, F. D. Souza, F. S. Nakutz, E. A. Roman, L. A. Velloso, A. S. Torsoni and M. A. Torsoni (2011). "Maternal high-fat feeding through pregnancy and lactation predisposes mouse offspring to molecular insulin resistance and fatty liver." J Nutr Biochem.

Belgardt, B. F. and J. C. Bruning (2010). "CNS leptin and insulin action in the control of energy homeostasis." Ann N Y Acad Sci 1212: 97-113.

Blundell, J. E. (1977). "Is there a role for serotonin (5hydroxytryptamine) in feeding?" Int J Obes 1(1): 15-42.

Blundell, J. E. (1984). "Serotonin and appetite." Neuropharmacology 23(12B): 1537-1551.

Bonavera, J. J., M. G. Dube, P. S. Kalra and S. P. Kalra (1994). "Anorectic effects of estrogen may be mediated by decreased neuropeptide-Y release in the hypothalamic paraventricular nucleus." Endocrinology 134(6): 23672370 .

Bruinvels, A. T., J. M. Palacios and D. Hoyer (1993). "Autoradiographic characterisation and localisation of 5HT1D compared to 5-HT1B binding sites in rat brain." Naunyn Schmiedebergs Arch Pharmacol 347(6): 569-582.

Christensen, H. N., L. M. Albritton, D. K. Kakuda and C. L. MacLeod (1994). "Gene-product designations for amino acid transporters." J Exp Biol 196: 51-57.

Churruca, I., M. P. Portillo, L. Casis, A. Gutierrez, M. T. Macarulla and E. Echevarria (2008). "Effects of fluoxetine administration on hypothalamic melanocortin system in obese Zucker rats." Neuropeptides 42(3): 293299.

Collaboration, N. C. D. R. F. (2017). "Worldwide trends in body-mass index, underweight, overweight, and obesity from 1975 to 2016: a pooled analysis of 2416 populationbased measurement studies in 128.9 million children, adolescents, and adults." Lancet 390(10113): 2627-2642.

Curzon, G., J. Friedel and P. J. Knott (1973). "The effect of fatty acids on the binding of tryptophan to plasma protein." Nature 242(5394): 198-200.

Dourish, C. T. (1995). "Multiple serotonin receptors: opportunities for new treatments for obesity?" Obes Res 3 Suppl 4: 449S-462S.

Dragunow, M. and R. Faull (1989). "The use of c-fos as a metabolic marker in neuronal pathway tracing." $\mathrm{J}$ Neurosci Methods 29(3): 261-265.

Dryden, S., M. Brown, P. King and G. Williams (1999). "Decreased plasma leptin levels in lean and obese Zucker rats after treatment with the serotonin reuptake inhibitor fluoxetine." Horm Metab Res 31(6): 363-366.

Erickson, J. D., M. K. Schafer, T. I. Bonner, L. E. Eiden and E. Weihe (1996). "Distinct pharmacological properties and distribution in neurons and endocrine cells of two isoforms of the human vesicular monoamine transporter." Proc Natl Acad Sci U S A 93(10): 5166-5171.
Estadella, D., L. M. Oyama, A. R. Damaso, E. B. Ribeiro and C. M. Oller Do Nascimento (2004). "Effect of palatable hyperlipidic diet on lipid metabolism of sedentary and exercised rats." Nutrition 20(2): 218-224.

Fam, B. C., M. J. Morris, M. J. Hansen, M. Kebede, S. Andrikopoulos, J. Proietto and A. W. Thorburn (2007). "Modulation of central leptin sensitivity and energy balance in a rat model of diet-induced obesity." Diabetes Obes Metab 9(6): 840-852.

Ferro Cavalcante, T. C., J. M. Lima da Silva, A. A. da Marcelino da Silva, G. S. Muniz, L. M. da Luz Neto, S. Lopes de Souza, R. Manhaes de Castro, K. M. Ferraz and E. do Nascimento (2013). "Effects of a westernized diet on the reflexes and physical maturation of male rat offspring during the perinatal period." Lipids 48(11): 1157-1168.

Fidalgo, S., D. K. Ivanov and S. H. Wood (2012). "Serotonin: from top to bottom." Biogerontology 14(1): 21-45.

Fuller, R. W. and D. T. Wong (1990). "Serotonin uptake and serotonin uptake inhibition." Ann N Y Acad Sci 600: 6878; discussion 79-80.

Gray, R. W. and S. J. Cooper (1996). "d-fenfluramine's effects on normal ingestion assessed with taste reactivity measures." Physiol Behav 59(6): 1129-1135.

Guan, X. M. and W. J. McBride (1989). "Serotonin microinfusion into the ventral tegmental area increases accumbens dopamine release." Brain Res Bull 23(6): 541547.

Gutierrez, A., G. Saracibar, L. Casis, E. Echevarria, V. M. Rodriguez, M. T. Macarulla, L. C. Abecia and M. P. Portillo (2002). "Effects of fluoxetine administration on neuropeptide $y$ and orexins in obese zucker rat hypothalamus." Obes Res 10(6): 532-540.

Heisler, L. K., E. E. Jobst, G. M. Sutton, L. Zhou, E. Borok, Z. Thornton-Jones, H. Y. Liu, J. M. Zigman, N. Balthasar, T. Kishi, C. E. Lee, C. J. Aschkenasi, C. Y. Zhang, J. Yu, O. Boss, K. G. Mountjoy, P. G. Clifton, B. B. Lowell, J. M. Friedman, T. Horvath, A. A. Butler, J. K. Elmquist and M. A. Cowley (2006). "Serotonin reciprocally regulates melanocortin neurons to modulate food intake." Neuron 51(2): 239-249.

Heisler, L. K., R. B. Kanarek and A. Gerstein (1997). "Fluoxetine decreases fat and protein intakes but not carbohydrate intake in male rats." Pharmacol Biochem Behav 58(3): 767-773.

Heisler, L. K., R. B. Kanarek and B. Homoleski (1999). "Reduction of fat and protein intakes but not carbohydrate intake following acute and chronic fluoxetine in female rats." Pharmacol Biochem Behav 63(3): 377-385.

Iniguez, S. D., B. L. Warren and C. A. Bolanos-Guzman (2010). "Short- and long-term functional consequences of fluoxetine exposure during adolescence in male rats." Biol Psychiatry 67(11): 1057-1066.

Kirk, S. L., A. M. Samuelsson, M. Argenton, H. Dhonye, T. Kalamatianos, L. Poston, P. D. Taylor and C. W. Coen (2009). "Maternal obesity induced by diet in rats permanently influences central processes regulating food intake in offspring." PLoS One 4(6): e5870.

Lauzurica, N., L. Garcia-Garcia, J. A. Fuentes and M. Delgado (2013). "Hypophagia and induction of serotonin transporter gene expression in raphe nuclei of male and female rats after short-term fluoxetine treatment." J Physiol Biochem 69(1): 69-74. 
Leibowitz, S. F. and J. T. Alexander (1998). "Hypothalamic serotonin in control of eating behavior, meal size, and body weight." Biol Psychiatry 44(9): 851-864.

Li, Q., N. A. Muma and L. D. van de Kar (1996). "Chronic fluoxetine induces a gradual desensitization of 5-HT1A receptors: reductions in hypothalamic and midbrain $\mathrm{Gi}$ and $\mathrm{G}(\mathrm{o})$ proteins and in neuroendocrine responses to a 5HT1A agonist." J Pharmacol Exp Ther 279(2): 10351042.

Lopez, J. F., D. T. Chalmers, D. M. Vazquez, S. J. Watson and H. Akil (1994). "Serotonin transporter mRNA in rat brain is regulated by classical antidepressants." Biol Psychiatry 35(4): 287-290.

Lowe, M. R. and M. L. Butryn (2007). "Hedonic hunger: a new dimension of appetite?" Physiol Behav 91(4): 432439.

Matsuo, H., S. Tsukada, T. Nakata, A. Chairoungdua, D. K. Kim, S. H. Cha, J. Inatomi, H. Yorifuji, J. Fukuda, H. Endou and Y. Kanai (2000). "Expression of a system L neutral amino acid transporter at the blood-brain barrier." Neuroreport 11(16): 3507-3511.

Mennigen, J. A., E. A. Harris, J. P. Chang, T. W. Moon and V. L. Trudeau (2009). "Fluoxetine affects weight gain and expression of feeding peptides in the female goldfish brain." Regul Pept 155(1-3): 99-104.

Messina, M. M., G. Boersma, J. M. Overton and L. A. Eckel (2006). "Estradiol decreases the orexigenic effect of melanin-concentrating hormone in ovariectomized rats." Physiol Behav 88(4-5): 523-528.

O'Dell, L. E. and L. H. Parsons (2004). "Serotonin1B receptors in the ventral tegmental area modulate cocaineinduced increases in nucleus accumbens dopamine levels." J Pharmacol Exp Ther 311(2): 711-719.

Oliva, J. M., L. Uriguen, S. Perez-Rial and J. Manzanares (2005). "Time course of opioid and cannabinoid gene transcription alterations induced by repeated administration with fluoxetine in the rat brain." Neuropharmacology 49(5): 618-626.

Pardridge, W. M. and G. Fierer (1990). "Transport of tryptophan into brain from the circulating, albumin-bound pool in rats and in rabbits." J Neurochem 54(3): 971-976.

Parsons, L. H. and J. B. Justice, Jr. (1993). "Serotonin and dopamine sensitization in the nucleus accumbens, ventral tegmental area, and dorsal raphe nucleus following repeated cocaine administration." J Neurochem 61(5): 1611-1619.

Pratt, W. E., K. Blackstone, M. E. Connolly and M. J. Skelly (2009). "Selective serotonin receptor stimulation of the medial nucleus accumbens causes differential effects on food intake and locomotion." Behav Neurosci 123(5): 1046-1057.
Ruddick, J. P., A. K. Evans, D. J. Nutt, S. L. Lightman, G. A. Rook and C. A. Lowry (2006). "Tryptophan metabolism in the central nervous system: medical implications." Expert Rev Mol Med 8(20): 1-27.

Sghendo, L. and J. Mifsud (2012). "Understanding the molecular pharmacology of the serotonergic system: using fluoxetine as a model." J Pharm Pharmacol 64(3): 317-325.

Siegel, A. and J. Douard (2011). "Who's flying the plane: serotonin levels, aggression and free will." Int J Law Psychiatry 34(1): 20-29.

Simansky, K. J. (1996). "Serotonergic control of the organization of feeding and satiety." Behav Brain Res 73(1-2): 37-42.

Smith, Q. R., S. Momma, M. Aoyagi and S. I. Rapoport (1987). "Kinetics of neutral amino acid transport across the blood-brain barrier." J Neurochem 49(5): 1651-1658.

Srinivasan, M., S. D. Katewa, A. Palaniyappan, J. D. Pandya and M. S. Patel (2006). "Maternal high-fat diet consumption results in fetal malprogramming predisposing to the onset of metabolic syndrome-like phenotype in adulthood." Am J Physiol Endocrinol Metab 291(4): E792-799.

Tsujii, S., Y. Nakai, J. Fukata, T. Koh, H. Takahashi, T. Usui and H. Imura (1986). "Effects of food deprivation and high fat diet on opioid receptor binding in rat brain." Neurosci Lett 72(2): 169-173.

Vucetic, Z., J. L. Carlin, K. Totoki and T. M. Reyes (2012). "Epigenetic dysregulation of the dopamine system in dietinduced obesity." J Neurochem 120(6): 891-898.

Wade, P. R., J. Chen, B. Jaffe, I. S. Kassem, R. D. Blakely and M. D. Gershon (1996). "Localization and function of a 5HT transporter in crypt epithelia of the gastrointestinal tract." J Neurosci 16(7): 2352-2364.

WANG, Y., C. MONTEIRO and B. M. POPKIN (2002). "Trends of obesity and underweight in older children and adolescents in the United States, Brazil, China, and Russia." Am J Clin Nutr 75(6): 971-977.

Weiss, G. F., N. Rogacki, A. Fueg, D. Buchen, J. S. Suh, D. T. Wong and S. F. Leibowitz (1991). "Effect of hypothalamic and peripheral fluoxetine injection on natural patterns of macronutrient intake in the rat." Psychopharmacology (Berl) 105(4): 467-476.

Yadav, V. K., F. Oury, K. F. Tanaka, T. Thomas, Y. Wang, S. Cremers, R. Hen, A. Krust, P. Chambon and G. Karsenty (2011). "Leptin-dependent serotonin control of appetite: temporal specificity, transcriptional regulation, and therapeutic implications." J Exp Med 208(1): 41-52.

Yoshimoto, K. and W. J. McBride (1992). "Regulation of nucleus accumbens dopamine release by the dorsal raphe nucleus in the rat." Neurochem Res 17(5): 401-407. 